\title{
Depression and quality of life in patients with diabetes
}

\author{
Papadopoulou L., ${ }^{1, *}$, Papoulia . $^{2}$ \\ ${ }^{1}$ General Hospital of Thessaloniki "Agios Pavlos”, Greece \\ ${ }^{2}$ General Hospital of Thessaloniki “Agios Dimitrios”, Greece
}

Email address:

lemontritsa@yahoo.gr (Papadopoulou L.), fotinipapoulia@yahoo.gr (Papoulia F.)

\section{To cite this article:}

Papadopoulou L., Papoulia F.. Depression and Quality of Life in Patients with Diabetes. American Journal of Nursing Science. Special Issue: Mental Health Care: Aspects, Challenges and Perspectives. Vol. 4, No. 2-1, 2015, pp. 88-91. doi: 10.11648/j.ajns.s.2015040201.26

\begin{abstract}
Diabetes mellitus is a syndrome of disorders where the patient follows a personalized insulin therapy and adjusts his diet and way of life. The purpose of this study is to describe the correlation of depression and anxiety experienced by patients with diabetes compared with their quality of life. Diabetic patient must make significant changes in daily life and lifestyle and has a greater need for social and psychological support, due to permanent complications and negative emotions experienced.
\end{abstract}

Keywords: Diabetes, Quality of Life and Depression

\section{Introduction}

Diabetes is a major health problem worldwide. The complications of this disease often causes serious health problems [1].The challenge for the diabetic patient is to adopt and maintain certain basic lifestyle changes, aimed to prevent long-term complications, maintaining blood glucose levels as possible physiologically[2].According to the World Health Organization (WHO, 2005),in2002 diabetes was a worldwide 11 th cause of death, with rate $1,7 \%$ of the total population [ 3 , 4].According to an estimate of the organization, the year 2030, provided increase its position as a major cause of death and ranking in 6th place, with almost double the rate of. Latest data from the Ministry of Health and Human Services of the United States of America, confirming the above case [5].Diabetes describes a set of complex disorders, which a common characteristic, the disturbances in metabolism and glucose utilization due to dysfunction of beta cells of the pancreas [4].The pathogenesis of diabetes mellitus has a common denominator, the insulin deficiency, which may be caused by reduced insulin secretion, due to defective secretion mechanism of beta-cells, in response to physiological stimuli and in the production and release of a biologically inactive form of insulin. The disease is treated through a team approach, which empowers the patient to confront and successfully manage the disease [6].On the other hand, the concept of quality of life associated with the subjective perception of people about their lives, within specific cultural characteristics and a specific system of social values. Within this framework, the people live according to their own goals, expectations, standards and concerns, which often create anxiety and therefore depression, with negative effects on quality of their lives [7].This paper aims to describe all the factors associated with diabetes and the effects of anxiety and depression experienced by patients in their quality of life.

\section{Material and Methods}

The material of this study is consisted of recent articles on the topic were found mainly in electronic databases such as Medline and the Greek Academic Libraries Link (HEALLink), with keywords: diabetes, quality of life and depression.

\section{Classification of Types of Diabetes}

The pathogenesis of diabetes mellitus has a common denominator, the insulin deficiency, which may be caused by reduced insulin secretion, due to defective secretion mechanism of beta-cells, in response to physiological stimuli and in the production and release of a biologically inactive form of insulin [6]. In the absence of insulin, the glucose is absorbed from the gastrointestinal tract cannot be used by the tissues, cannot be converted to glycogen, resulting in hyperglycemia [5, 7].Diabetes and its various forms are introduced to the international classification of diseases and causes of death, ICD - 10.The classification regards the codes E10-E14. According to Karamitsos (2009)concerns the 
E10 or insulin dependent diabetes(IDDM - Insulin Depended Diabetes Mellitus), the E11 or noninsulin(NIDDM - Non Insulin Depended Diabetes Mellitus), the E12 which is associated with improper $\operatorname{diet}(\mathrm{MRDM}$ Mal nutrition Related Diabetes Mellitus), the E13 including third types of diabetes(Other specified diabetes mellitus) and finally the E14 that contains undefined forms of diabetes (Unspecified diabetes mellitus) [8].

\section{Diabetes Treatment}

Unfortunately, there is no cure for diabetes. The aim is to keep the prices of glucose and lipids in normal levels and these factors be controlled as strictly as possible to prevent complications. The protocol for the control of diabetes is individualized and depends on the type of diabetes the patient has, age, state of health, the ability to follow the recommended treatment, the acceptance of responsibility to control the disease and the existence of other factors [7, 8].Medication for diabetes based primarily on insulin in combination with, or without, hypoglycemic drugs orally. At the beginning of therapy, the medication administered in the lowest effective dose, which is gradually increased every 1-2 weeks until the patient is approaching an acceptable level of glycemic control or until reach the maximum dose. If this maximum dose may not achieve sufficient glycemic control should be used another antidiabetic agent orally. The insulin therapy is indicated when the levels of glucose in the blood cannot be brought under control after using two or three different antidiabetic agents [2].Today are available several methods of insulin administration, apart from the classic subcutaneous injections. The continuous subcutaneous administration of basic insulin dose with further increase of the administered insulin during mealtime sis the effective method to control the level of glucose in relation to the treatment regimen of multiple insulin injections [9].

\section{Quality of Life and Diabetes Mellitus}

The importance of evaluating quality of life in patients with chronic diseases is widely accepted by the scientific community. In chronic diseases both the disease itself and therapeutic strategies can lead to negative symptoms and a variety of difficulties in the way of life of patients and their families $[10,11]$.In particular, patients with diabetes face the disease every day, all day, since it is necessary to take countless decisions in their attempt to reach the non-diabetic metabolic levels. Treatment, like insulin, may interfere with either positive (lowering high blood sugar levels), or negative (raise low blood sugar levels) the quality of life of diabetic patients $[10,12]$.The psychosocial cost of diabetes for patients is very high and often affects the self-care behaviors, controlling disease and other complications, and quality of life. The depressive symptoms, for example, are powerful predictors, compared with changes in metabolic measurements [12, 13].Additionally, it has been shown that psychosocial factors may act protective and assist in good care and management of diabetes. The objectives regarding the assessment of psychosocial health and quality of life of people suffering from diabetes include:the recognition of patients who develop symptoms of depression or anxiety, the evaluation of new therapeutic techniques, considering the psychosocial advantages and disadvantages and the recognition of possible dissatisfaction treatment or other aspects of care benefits [14].The effort in each case refers to the provision of health services aimed in maintaining or improving the quality of life of diabetic patients [15].Diabetes mellitus is one test for the patient but also for his family, because of the chronicity and uncertainty regarding the course and outcome of the disease. Although each disease presents different clinical symptoms there are many common problems in all chronic patients. Usually chronic disease affects catalytic in basic functions of the individual, such as communication, sociability and self-care $[15,16]$.The chronicity of the disease diabetes can be one of the main aggravating factors, exhausting psychological and physical the individual weakened his forces and reduces his mental stamina. This leads to adverse effects not only on health but also in his attempt, for compliance to treatment [17].Concluding, all the above, are undermining the quality of life of the diabetic patient. Consequently, various cognitive and emotional responses generated as a result of stress and anxiety about the outcome of the disease in daily life and quality of life. The knowledge and skills acquired by the person, and his experiential experience, are the key element to improve the personal well-being. Of the most important factors that aggravated the quality of life of people with diabetes is depression and anxiety due to the self-regulation of diabetes, its complications and chronicity of the disease [18].

\section{Depression and Diabetes Mellitus}

The negative mood caused by diabetes to the patient, combined with the factor of chronicity of the disease and the difficulty involved in self-regulation, creates prolonged stress, and by extension, huge psychological burden, as a result this affect their quality of life. The above concern is exacerbated by the stressful nature of the disease and its impact in daily routine of the patient [19, 20].Anxiety and stress usually associated with the fact that the disease is incurable followed by various unpleasant social impact on the job, in the marital life and in social life and substantial changes in everyday life. The chronic complications of the disease, nephropathy, neuropathy, diabetic foot and other side effects, instruct the person with additional stress [21, 22].The adaptation to the demands of the illness, such as impossibility of maintaining the level of glucose to the normal range , and the need for continuous control of diet and insulin therapy programs, affecting significantly the quality of life of people. The weakness of the regulation of clinical parameters to control the course of the disease, involves negative implications on self-image and self-esteem [22].Similar negative effects on the quality of life of individuals have frequent episodes of 
hypoglycemia. The patients affected most are possessed by fear of a hypoglycemic episode. The unexpected episodes of nocturnal hypoglycemia, which occurs when the patient is alone, can cause feelings of threat and inability to regulate his life. This fact, in front of people, can cause strong feelings of shame and guilt, which contribute to reducing of social interaction and eventually isolate the patient. Each patient, however, must be familiarized with lifelong threat of hypoglycemia, which can cause chronic stress and psychosomatic disorders [23, 24].The fear of some upcoming hypoglycemic episode is one of the major stressors factors. The activation of the stress system mobilizes a series of behavioral and peripheral responses due to actuation of the axishypothalamic-pituitary-adrenaland the sympathetic nervous system, such as increasing of glucose, heart rate, blood pressure and other parameters. Stress enhances alertness and promotes certain behaviors of the individual, while simultaneously inhibits the natural functions such as the desire of food intake and reproduction. In an intense stressful situation appears more stressful symptoms such as hyperactivity to stimuli and the adoption of a "frozen" behavior [25].On the other hand, the probability of depression is more common in people with inadequate glycemic control in people who have experienced the first symptoms of complications of the disease and those who have a reduced ability in the various treatment programs disease. Significant correlation seen between depression symptoms in relation to the perception of the assessment of their health and their quality of life, self-care and glycemic control. $[4,26]$.Especially for young people, the arrival of the disease on their lives is a traumatic event, but also a reference point in their lives. Child refers chronologically in the "before" and "after" diabetes with nostalgia for the "before" period and melancholy for "after" [22, 27].The sense of risk of disease and the fact that there is no definitive treatment is a powerful reinforcing agent for the appearance of chronic depression.At the same time, the idea that diabetes interferes to the wishes, expectations and occupations recommends reinforcing element for the development of depressive semiology in diabetic patients. The threat associated with the psychological impact of diabetes in confidence, happiness, or satisfaction with life is able to significantly predict depression which may experience people with diabetes [19, 22].Psychotherapy and the use of mild psychiatric drugs can relieve patients, but also significantly improve their euphoria, bring back normal situation of sleeping and eating habits, which are important in diabetes. Also to improve the physical condition and patient compliance, to treatment. On the other hand, the purpose of psychotherapy is to accept the specificity and diversity compared to other people. Approximately, early diagnosis and treatment of depression only occurs in $25 \%$ of cases [25].

\section{Social Care and Diabetes Mellitus}

Social support is a very important chapter in the life of diabetic patients, and generally for people suffering from chronic diseases such as diabetes. [19, 22].The availability of social support plays a major role in the ability of the psychosocial adjustment of patient towards to chronic disease because it contributes to the reconstruction of thoughts and finding solutions to the immediate crisis response. [15, 26].People usually turn to the wider social environment to seek available assistance in order to cope with the stressful events of their disease. The perception of patient, that available social support for his problems, can work therapeutically not only short term but long term, making him feel real support from close to him persons, even in periods of depression [16,25].Particularly useful is the existence of a supportive network of young people suffering from insulin-dependent diabetes .In a sensitive developmental period of his life the young person comes for the first time confronted with the particular demands of diabetes, which require a certain degree of self-care. The patient must be adapted to feeding standards and treatment that cannot be implemented adequately, because of cognitive deficits of the early and middle childhood. [15.28].Despite the training of health services, the family shares of responsibility on the management of the disease, because enough people find it difficult to cope with the requirements. Usually, the adolescent with insulin dependent diabetes feels that he is different and in his attempt to conceal the existence of chronic disease leaves the setup programor presents isolation tendencies, so consequently hampered its integration into the group of his peers. [23, 24].The fear of social stigma of course affects the family. Unfortunately many times, parents fear for not accepting the child because of chronic illness and this fact removes the family from the wider family and social environment. The consequence of all this is to reduce the possibility of providing support, when it is available [29].It has been observed that a young diabetic patient considers the family as the most important adjustment factor in the disease. This is due to the fact that the family covers the priority needs and the necessary emotional support is required [30].It is particularly important to consider that the sickness of a family member is substantially affects the whole family. In particular, the family is experiencing a crisis and begins to observed disturbances in its cohesion. Nevertheless, the family should focus on the skills and abilities of the child thus shifting the focus of self-care to existing features [16].

\section{Conclusions}

For a diabetic patient is important to maintain an often demanding lifestyle. Also important is the direct and indirect impact of various negative psychological and social variables in physical health. The long-lasting and effective treatment of the disease, however, can only be achieved with the cooperation of many experts from the field of biomedical sciences and the field of behavioral science, provided suitable training of expert staff as doctor, nurse, psychologist, social worker etc. [20].The most important aim of care professionals in diabetes lies in supporting patients to make 
appropriate self-care behaviors and patient education on these behaviors [30].Regarding the psychological interventions are proven to have a positive effect both on physical and mental well-being of the patient [31].

\section{References}

[1] Dewit S. Medicine Surgical Nursing. Concepts and Practice, 2nd Volume, Litsas Medical Publishing, Athens, 2009.

[2] Tsiftsoglou A. Molecular and Clinical Pharmacology - Basic principles and mechanisms of action of drugs, 2nd Volume, University Studio Press Publishing. Thessaloniki, 2001.

[3] Colin D M, Dejan L. Updated projections of global mortality and burden of disease, 2002-2030: Data sources, methods and results. Evidence and Information for Policy Working Paper. World Health organization, October2005. Available at: www.who.com

[4] Papadopoulos A, Oikonomakis E, Kontodimopoylos N, Fridas A \& Niakas D. Assessment of quality of health-related life diabetic type II patients. Archives of Greek Medicine, 2007, $24: 66-74$

[5] Centers for Disease Control and Prevention.2011; National Diabetes Fact Sheet. Atlanta, GA: Centers for Disease Control and Prevention, US Department of Health and Human Services. [Online]. Available at: http://www.cdc.gov/. [accessed16.9.13].

[6] Harvey R. A., Champe P. C. Pharmacology, 3d Edition, Parisianou Publishing, Athens, 2007.

[7] Kavoura M., Kiriopoulos G., Geitona M., Vandorou Chr. Quality of life, Janssev-Cilag Publishing, Athens, 2003.

[8] Karamitsos D Diabetology: Theory and practice in the treatment of diabetes, Siokis Publishing,2nd Edition, Athens, 2009.

[9] Katsilampros N. Diadetes Melitus, Arxipelagos. Publishing, Athens, 2007.

[10] Bott, U., Mühlhauser, I., Overmann, H., \&Berger, M. Validation of a diabetes-specific quality-of-life scale for patients with type 1 diabetes. Diabetes Care, 2003;21(5), 75769.

[11] Kourkouta L, Barsamidis K, Lavdaniti M. Communication skills during the clinical examination of the patients. Progress in Health Sciences,2013; 3(1) 119-122

[12] Peyrot, M., \& Rubin, R.R. Behavioral and Psychosocial Interventions in Diabetes. DiabetesCare, 2007; 30:2433-2440

[13] Rosenthal, M.J., Fajardo, M., Gilmore, S., Morley, J.E., \& Nabiloff, B.D. Hospitalization and mortality of diabetes in older adults: a three-year prospective study. Diabetes Care,2004;21: 231-235.
[14] Calvert, M.J., \& Freemantle, N. Use of health-related quality of life in prescribing research. Part I: Why evaluate healthrelated quality of life? Journal of Clinical Pharmacy and Therapeutics,2003; 28: 513-521.

[15] Tsaousoglou A, Koukourikos K. Quality and health services. Stigma,2007; 15(1):18-24.

[16] Sapountzi - Krepia D. Chronic Disease and Nursing care- A holistic approach, Hellin Publishing,Athens,2004.

[17] Pita R, Grigoriadou E, Marina E, Kouvatsou Z. Quality of life and diabetes mellitus type 1. Greek Diabetes Chronicles, 2006;19 (4): 282-294.

[18] Theofilou P. The quality of life in health. e-magazine Science and Technology, 2010; 43-50.

[19] Papathanasiou I, Sklavou M, Kourkouta L. Holistic nursing care: theories and perspectives. American Journal of Nursing Science, 2013;2(1): 1-5.

[20] Karadimas, Health Psychology: Theory and Clinical Practice. Athens, 2005

[21] Kourkouta L Papathanasiou. V I. Communication in Nursing Practice. MaterSociomed, 2014; 26(1): 66-68.

[22] Grigoriadou E. Functionality and quality of life in people with diabetes type 1. Aristotle University of Thessaloniki, Department of Psychology, Thessaloniki, 2005.

[23] Kourkouta L, Hadjidimitriou H, Dalagozi P. The Confidentiality of People with Mental Health Problems by the Nurses. ICN 22nd Quadrennial Congress. Copenhagen, June10-15, 2001, Abstracts, Vol.II,2001.

[24] Ouzounakis P, Chalkias Th. The confidentiality of «Medical Secrets» of Patients by the nursing staff. International Journal of Caring Sciences, 2009; 3(1):1-2.

[25] Wasserman L., Trifonova E. Diabetes Mellitus as a model of Psychosomatic and Somatopsychic Interrelations. The Spanish Journal of Psychology, 2006; 9(1), 75-85.

[26] Kourkouta L. Announcing sad news. Hospital Chronicles. 1994;56(1):60:63.

[27] Kourkouta L, Prokopiou E, Fratzana K, Amprahim S.E, Papastergiou K. Children with diabetes in school. Scientific Chronicles, 2012; $17(4)$ ): 203-208.

[28] Kourkouta L, Rarra A, Amprahim S. E,The diet of children in school age. Scientific Chronicles, 2013; 18(2) : 78 -82.

[29] Tsamatsiros G. The effect of insulin dependent diabetes mellitus in family functioning, Greek Diabetes Association. Diabetes News, 2003; vol 3.

[30] Wagner J., Abbott G., Syrretta L. Age related differences in individual quality of life domains in youth with type 1 diabetes. Health and Quality of Life Outcomes, 2004; 2:54.

[31] Gonder-Frederick, L., Cox, D. J., \& Ritterband, L. M. Diabetes and behavioral medicine: The second decade. Journal of Consulting and Clinical Psychology,2002; 70: 611-625. 